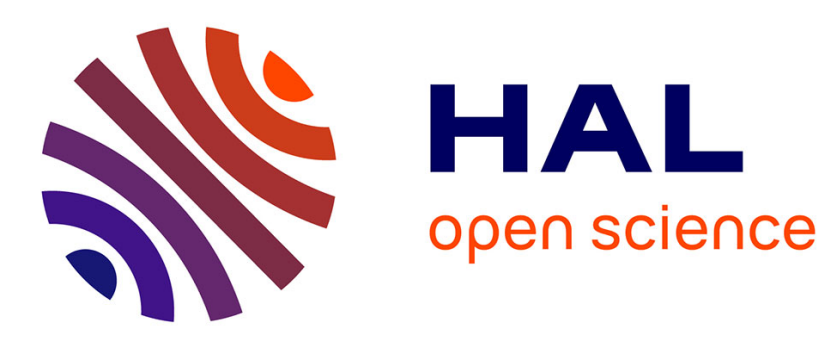

\title{
Exact discrete minimization for TV+L0 image decomposition models
}

Loïc Denis, Florence Tupin, X. Rondeau

\section{To cite this version:}

Loïc Denis, Florence Tupin, X. Rondeau. Exact discrete minimization for TV+L0 image decomposition models. Image Processing (ICIP), 2010 17th IEEE International Conference on, Sep 2010, Hong Kong SAR China. pp.2525 - 2528, 10.1109/ICIP.2010.5649204 • ujm-00985427

\section{HAL Id: ujm-00985427}

\section{https://hal-ujm.archives-ouvertes.fr/ujm-00985427}

Submitted on 29 Apr 2014

HAL is a multi-disciplinary open access archive for the deposit and dissemination of scientific research documents, whether they are published or not. The documents may come from teaching and research institutions in France or abroad, or from public or private research centers.
L'archive ouverte pluridisciplinaire HAL, est destinée au dépôt et à la diffusion de documents scientifiques de niveau recherche, publiés ou non, émanant des établissements d'enseignement et de recherche français ou étrangers, des laboratoires publics ou privés. 


\section{EXACT DISCRETE MINIMIZATION FOR TV+LO IMAGE DECOMPOSITION MODELS}

\section{Denis}

\author{
Observatoire de Lyon, CNRS CRAL, \\ UCBL, ENS de Lyon, Université de Lyon, \\ France
}

\author{
F. Tupin, X. Rondeau \\ Institut Telecom, Telecom ParisTech, \\ CNRS LTCI, \\ Paris, France
}

\begin{abstract}
Penalized maximum likelihood denoising approaches seek a solution that fulfills a compromise between data fidelity and agreement with a prior model. Penalization terms are generally chosen to enforce smoothness of the solution and to reject noise. The design of a proper penalization term is a difficult task as it has to capture image variability. Image decomposition into two components of different nature, each given a different penalty, is a way to enrich the modeling. We consider the decomposition of an image into a component with bounded variations and a sparse component. The corresponding penalization is the sum of the total variation of the first component and the L0 pseudo-norm of the second component. The minimization problem is highly non-convex, but can still be globally minimized by a minimum s-t-cut computation on a graph. The decomposition model is applied to synthetic aperture radar image denoising.
\end{abstract}

Index Terms - denoising, discrete minimization, graphcuts, synthetic aperture radar.

\section{INTRODUCTION}

Image denoising has been considered in the seminal work of Rudin, Osher and Fatemi (ROF) [1] as the decomposition of an image into a component with bounded variations (BV), and a noise component. The noise is removed by minimizing the total variation (TV) of the BV component, subject to a data fidelity constraint. The popularity of TV-based denoising comes from its ability to preserve sharp edges in the denoised image (i.e., in the BV component).

It has long been noted that ROF model leads to "cartoonlike" images. This can be better understood by considering ROF in a Bayesian framework. Data fidelity expresses the likelihood while total variation models the prior on the denoised image. Piecewise constant images have low total variation, and are therefore given a high prior probability. TV prior therefore biases the solution towards piecewise constant (i.e., "cartoon") images. This is often considered a problem, sometimes referred to as a staircasing effect [2].

This work has been funded by DGA under contract REI 2008.34.0042.
Meyer proposed to take advantage of this effect to perform a decomposition of an image into its geometrical (socalled "structure") and textural components. To model the prior of textures as highly oscillating, he suggested the use of the $G$-norm [3]. Since that work, several image decomposition models have been studied in the literature $[4,5,6,7]$. In some approaches, the focus is put on the decomposition of a noiseless image into two parts. In others, the image is decomposed into two parts plus a noise component. We follow the latter approach in this paper.

We consider the restoration of an image formed by the sum of a piecewise constant component and by some isolated pixels. Such a model can for example be applied to synthetic aperture radar (SAR) images in which the scene can often be considered as a mix of homogeneous regions and point-like strong scatterers. The restoration of the two components can be obtained by minimizing the sum of TV and LO, as discussed in section 2. The minimization problem to handle is challenging: it is combinatorial due to the L0 term. The specific form of the energy (namely, the separability of the nonconvex part) can be exploited to design an algorithm for exact discrete minimization. The algorithm, described in section 3 , is based on a minimum s-t-cut computation on a graph. We illustrate the method on numerical experiments and SAR data in section 4.

\section{THE TV+LO DECOMPOSITION MODEL}

We describe the problem of the recovery of a scene $\boldsymbol{u}$ from an observed image $\boldsymbol{v}$ corrupted by noise. Images are considered sampled and quantized. The scene decomposition model is introduced in the following paragraph. Then, noise modeling is described. Finally, the TV+L0 minimization problem is stated.

\subsection{Scene decomposition model}

We consider scenes $\boldsymbol{u}$ that can be decomposed as the sum of two terms: $\boldsymbol{u}=\boldsymbol{u}_{\mathrm{BV}}+\boldsymbol{u}_{\mathrm{S}}$, where $\boldsymbol{u}_{\mathrm{BV}}$ is a component with low total variation (i.e., close to piecewise constant), and $\boldsymbol{u}_{\mathrm{S}}$ is a sparse component (i.e., with all pixels but a few equal to zero). 
The total variation of component $\boldsymbol{u}_{\mathrm{BV}}$ can be approximated $^{1}$ by $\operatorname{TV}\left(\boldsymbol{u}_{\mathrm{BV}}\right)=\sum_{(k, l)} w_{k, l}\left|u_{\mathrm{BV} k}-u_{\mathrm{BV} l}\right|$, where $(k, l)$ denotes a clique (i.e., pair of neighboring pixels), and $w_{k, l}$ is a weight. The sparsity of component $\boldsymbol{u}_{\mathrm{S}}$ is defined by the value of the L0 pseudo-norm, i.e., the number of non-zero pixels of $\boldsymbol{u}_{\mathrm{S}}: \operatorname{LO}\left(\boldsymbol{u}_{\mathrm{S}}\right)=\sum_{k} 1-\delta\left(u_{\mathrm{S} k}\right)$.

By considering that the two components $\boldsymbol{u}_{\mathrm{BV}}$ and $\boldsymbol{u}_{\mathrm{S}}$ are statistically independent, the joint prior distribution $p\left(\boldsymbol{u}_{\mathrm{BV}}, \boldsymbol{u}_{\mathrm{S}}\right)$ can be modeled in the framework of Markov random fields by:

$$
\begin{aligned}
-\log p\left(\boldsymbol{u}_{\mathrm{BV}}, \boldsymbol{u}_{\mathrm{S}}\right) & =-\log p\left(\boldsymbol{u}_{\mathrm{BV}}\right)-\log p\left(\boldsymbol{u}_{\mathrm{S}}\right) \\
& =\beta_{\mathrm{BV}} \mathrm{TV}\left(\boldsymbol{u}_{\mathrm{BV}}\right)+\beta_{\mathrm{S}} \operatorname{LO}\left(\boldsymbol{u}_{\mathrm{S}}\right)
\end{aligned}
$$

\subsection{Image formation model}

We assume that the likelihood distribution that relates the noisy image $\boldsymbol{v}$ to the scene $\boldsymbol{u}$ is separable, i.e., can be written as a product of the likelihood at each pixel $k$ :

$$
p(\boldsymbol{v} \mid \boldsymbol{u})=\prod_{k} p\left(v_{k} \mid u_{k}\right)
$$

We therefore consider no mixing (such as that caused by blurring) or noise correlation in our image formation model.

If the noise is additive, the image $\boldsymbol{v}$ can be written as $\boldsymbol{v}=$ $\boldsymbol{u}_{\mathrm{BV}}+\boldsymbol{u}_{\mathrm{S}}+\boldsymbol{n}$, with $\boldsymbol{n}$ a noise component. In the case of multiplicative noise, like speckle noise occurring in coherent imaging technique such as SAR, the image writes $\boldsymbol{v}=\left(\boldsymbol{u}_{\mathrm{BV}}+\right.$ $\left.\boldsymbol{u}_{\mathrm{S}}\right) \times \boldsymbol{n}$. Note that for some noise distributions (e.g., Poisson noise, or Rice distribution), no such relation exist.

The likelihood distribution may depend on each term of the scene decomposition $p\left(v_{k} \mid u_{\mathrm{BV} k}, u_{\mathrm{S} k}\right)$, as will be discussed in section 4 .

\subsection{Energy minimization problem}

Starting from an observed noisy image $\boldsymbol{v}$, the components $\boldsymbol{u}_{\mathrm{BV}}$ and $\boldsymbol{u}_{\mathrm{S}}$ can be estimated in the maximum a posteriori sense by solving the following minimization problem:

$$
\begin{gathered}
\left(\widehat{\boldsymbol{u}_{\mathrm{BV}}, \boldsymbol{u}_{\mathrm{S}}}\right)=\underset{\left(\boldsymbol{u}_{\mathrm{BV}}, \boldsymbol{u}_{\mathrm{S}}\right)}{\arg \min }-\log p\left(\boldsymbol{v} \mid \boldsymbol{u}_{\mathrm{BV}}, \boldsymbol{u}_{\mathrm{S}}\right)-\log p\left(\boldsymbol{u}_{\mathrm{BV}}, \boldsymbol{u}_{\mathrm{S}}\right) \\
=\underset{\left(\boldsymbol{u}_{\mathrm{BV}}, \boldsymbol{u}_{\mathrm{S}}\right)}{\arg \min } \\
\mathrm{D}\left(\boldsymbol{v}, \boldsymbol{u}_{\mathrm{BV}}, \boldsymbol{u}_{\mathrm{S}}\right)+\beta_{\mathrm{BV}} \mathrm{TV}\left(\boldsymbol{u}_{\mathrm{BV}}\right)+\beta_{\mathrm{S}} \operatorname{LO}\left(\boldsymbol{u}_{\mathrm{S}}\right)
\end{gathered}
$$

Given an input image $\boldsymbol{v}$ with $N$ pixels, minimization problem (3) has $2 N$ unknowns. The objective function is noncontinuous and highly non-convex in variable $\boldsymbol{u}_{\mathrm{S}}$ (possibly also in variable $\boldsymbol{u}_{\mathrm{BV}}$ depending on $\left.\mathrm{D}(\cdot)\right)$ and is not separable in variable $\boldsymbol{u}_{\mathrm{BV}}$ (the TV term induces a coupling between values at different sites). It is thus very challenging to solve.

\footnotetext{
${ }^{1}$ our choice of anistropic TV rather than isotropic TV will find its justification in the use of the discrete minimization technique described in section 3
}

We describe next section how the global minimum can still be found by exact discrete minimization via a combinatorial optimization technique based on a minimum s-t-cut computation.

\section{EXACT DISCRETE MINIMIZATION BY GRAPH-CUTS}

We show in the following paragraphs how to solve minimization problem (3) exactly among discrete images $\boldsymbol{u}_{\mathrm{BV}}$ and $\boldsymbol{u}_{\mathrm{S}}$. At each of their $N$ pixels, these images take values respectively in the discrete ordered sets $\left\{\alpha_{1}, \alpha_{2}, \cdots, \alpha_{m_{\mathrm{BV}}}\right\}$ and $\left\{\gamma_{1}, \gamma_{2}, \cdots, \gamma_{m_{\mathrm{S}}}\right\}$.

\subsection{Problem reformulation}

We begin by considering minimization problem (3) for $\boldsymbol{u}_{\mathrm{BV}}$ fixed. We write $\boldsymbol{u}_{\mathrm{S}}^{\star}\left(\boldsymbol{u}_{\mathrm{BV}}\right)$ the minimizer of one such restricted problem:

$$
\boldsymbol{u}_{\mathrm{S}}^{\star}\left(\boldsymbol{u}_{\mathrm{BV}}\right)=\underset{\boldsymbol{u}_{\mathrm{S}}}{\arg \min } \mathrm{D}\left(\boldsymbol{v}, \boldsymbol{u}_{\mathrm{BV}}, \boldsymbol{u}_{\mathrm{S}}\right)+\beta_{\mathrm{S}} \mathrm{LO}\left(\boldsymbol{u}_{\mathrm{S}}\right)
$$

It comes from the separability of the data fidelity and L0 terms (see our hypotheses discussed in paragraph 2.2) that the computation of $\boldsymbol{u}_{\mathrm{S}}^{\star}\left(\boldsymbol{u}_{\mathrm{BV}}\right)$ requires to solve $N$ mono-dimensional minimization problems:

$$
\left\{\begin{array}{rlrl}
u_{\mathrm{S} k}{ }^{\star}\left(u_{\mathrm{BV} k}\right)=u_{\mathrm{S} k}^{\dagger} & & \text { if }-\log p\left(v_{k} \mid u_{\mathrm{BV} k}, u_{\mathrm{S} k}^{\dagger}\right)+\beta_{\mathrm{S}} \\
& <-\log p\left(v_{k} \mid u_{\mathrm{BV} k}, u_{\mathrm{S} k}=0\right), \\
u_{\mathrm{S} k}{ }^{\star}\left(u_{\mathrm{BV} k}\right)=0 & & \text { otherwise; }
\end{array}\right.
$$

with $u_{\mathrm{S}_{k}}^{\dagger}=\arg \min _{u_{\mathrm{S}}}-\log p\left(v_{k} \mid u_{\mathrm{BV} k}, u_{\mathrm{S}_{k}}\right)$ either known in closed form, or numerically computed in $O\left(m_{\mathrm{S}}\right)$ operations in the worst case.

We can now reformulate minimization problem (3) into an equivalent problem involving only $\boldsymbol{u}_{\mathrm{BV}}$ :

$$
\begin{array}{r}
\underset{\boldsymbol{u}_{\mathrm{BV}}}{\arg \min } \underbrace{\mathrm{D}\left(\boldsymbol{v}, \boldsymbol{u}_{\mathrm{BV}}, \boldsymbol{u}_{\mathrm{S}}^{\star}\left(\boldsymbol{u}_{\mathrm{BV}}\right)\right)+\beta_{\mathrm{S}} \mathrm{LO}\left(\boldsymbol{u}_{\mathrm{S}}^{\star}\left(\boldsymbol{u}_{\mathrm{BV}}\right)\right)}_{(\mathrm{i})} \\
\underbrace{+\beta_{\mathrm{BV}} \mathrm{TV}\left(\boldsymbol{u}_{\mathrm{BV}}\right)}_{\text {(ii) }}
\end{array}
$$

The term (i) is of the form $\sum_{k} f_{k}\left(u_{\mathrm{BV}}\right)$ (i.e., it is separable), and (ii) is a convex term of the form $\sum_{(k, l)} g_{k l}\left(u_{\mathrm{BV} k}, u_{\mathrm{BV} l}\right)$ (i.e., involving only first order cliques).

Solving problem (6) gives $\boldsymbol{u}_{\mathrm{B} V}^{\star}$. The pair $\left(\boldsymbol{u}_{\mathrm{BV}}^{\star}, \boldsymbol{u}_{\mathrm{S}}^{\star}\left(\boldsymbol{u}_{\mathrm{BV}}^{\star}\right)\right)$ obtained is then a global minimizer of problem (3). We describe in next paragraph how problem (6) can be stated equivalently as a minimum s-t-cut computation on a graph.

\subsection{Graph representation}

Minimization problems of the form of problem (6), with a non-convex but separable discrete potential $f_{k}$ and a con- 
(a) Pixel grid

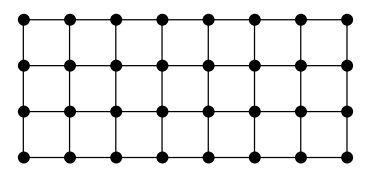

(c) Arc orientations and weights
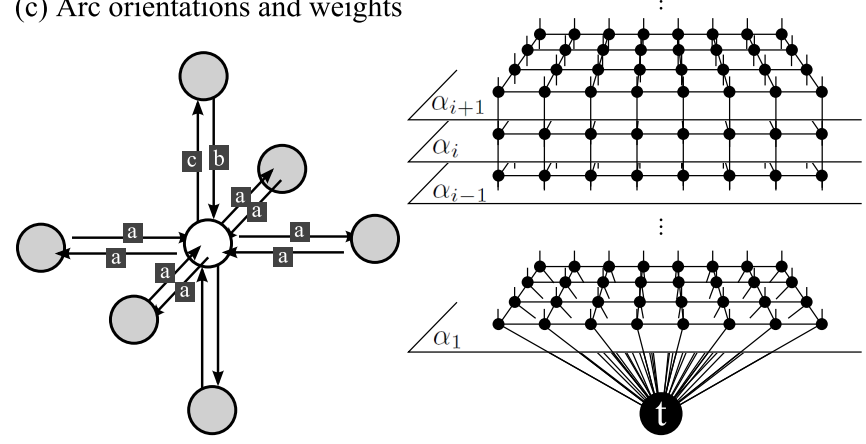

Fig. 1. Graph construction for discrete minimization.

vex discrete potential over first-order cliques $g_{k l}$ can be exactly solved by computing a minimum s-t-cut on a graph with $N \times m_{\mathrm{BV}}$ nodes $[8,9]$. We give here a quick overview of how to restate such a minimization problem as a min-cut computation.

We begin by giving a brief description of Ishikawa's graph construction [8]. As depicted in figure 1, the graph is a superimposition of layers of nodes. Each layer has $N$ nodes, one for each image pixel, and pairs of arcs connect neighboring nodes (the cliques of the underlying pixel grid shown in Fig.1(a)). The $m_{\mathrm{BV}}$ different layers represent each possible level of the discrete set $\left\{\alpha_{1}, \alpha_{2}, \cdots, \alpha_{m_{\mathrm{BV}}}\right\}$. Two special nodes (terminals) are added: a source $\mathrm{s}$ and a sink $\mathrm{t}$. Each node is also connected to its two counterparts in the next layer below and above. The first and last layers are connected respectively to the source and sink. Finally, a capacity is set to each arc, as depicted in Fig.1(c). The node corresponding to pixel $k$, located on the $i$-th layer, represents the value $u_{\mathrm{BV} k}=\alpha_{i}$. It is connected to its neighbors from the same layer with horizontal arcs with capacity $\mathbf{a}=\beta_{\mathrm{BV}} \cdot\left(\alpha_{i+1}-\alpha_{i}\right)$. The vertical arc going downstream (from source to sink) is given the weight $\mathbf{b}=f_{k}\left(\alpha_{i}\right)$. The vertical arc going upstream is given infinite cost $\mathbf{c}=\infty$.

With this construction, cuts with finite cost are in bijection with the set $\left\{\alpha_{1}, \alpha_{2}, \cdots, \alpha_{m_{\mathrm{BV}}}\right\}^{N}$ of all possible discrete images $\boldsymbol{u}_{\mathrm{BV}}$ (see [8]). The cost of each cut equals the value of the energy minimized in (6) for the corresponding image $\boldsymbol{u}_{\mathrm{BV}}$. Thus, the minimum cut gives the global minimizer $\boldsymbol{u}_{\mathrm{BV}}^{\star}$ of problem (6), from which we deduce $\boldsymbol{u}_{\mathrm{S}}^{\star}\left(\boldsymbol{u}_{\mathrm{BV}}^{\star}\right)$ to solve the initial image decomposition problem (3).

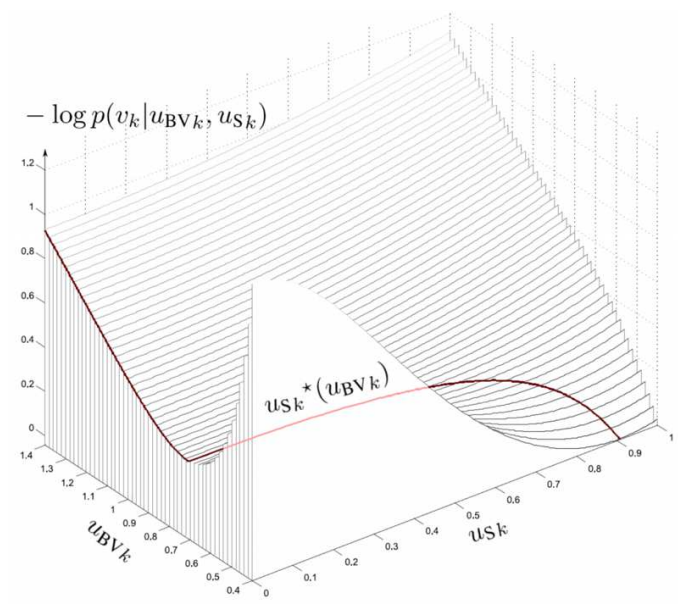

Fig. 2. Surface representation of Rice neg-log-likelihood (surface drawn for $v_{k}=1$ ).

\section{RESULTS}

We illustrate in the following paragraphs the application of the discrete minimization algorithm to decompose a SAR image into homogeneous regions and strong scatterers with the TV+L0 model.

\subsection{Model for $S A R$ images}

High and very high resolution SAR scenes are generally a mix of rather homogenous regions and point-like strong scatterers. These scatterers have strong contrast (amplitudes several orders of magnitude higher than that of the homogeneous background). Man-made structures (buildings, bridges, pylons, ... ) produce such strong echoes.

The observed SAR amplitude image $v$ is corrupted by speckle noise generated by the coherent summation of echoes coming from different scatterers. In a homogeneous region, noise follows Rayleigh distribution (Gamma distribution when considering intensity rather than amplitude). When a strong scatterer is present in a resolution cell, noise distribution is better described by a Rice distribution[10] (see Fig. 2):

$$
\begin{array}{r}
-\log p\left(v_{k} \mid u_{\mathrm{BV} k}, u_{\mathrm{S} k}\right)=\frac{v_{k}^{2}+u_{\mathrm{S} k}^{2}}{2 u_{\mathrm{BV} k}^{2}}+2 \log u_{\mathrm{BV} k} \\
-\log \mathrm{I}_{0}\left(\frac{v_{k} \cdot u_{\mathrm{S} k}}{u_{\mathrm{BV} k}^{2}}\right),
\end{array}
$$

with $\mathrm{I}_{0}$ modified Bessel function of the first kind. Note that the amplitude $u_{\mathrm{BV} k}$ must be strictly positive and that $u_{\mathrm{S} k}$ is either equal to zero (absence of strong scatterer) or strictly positive. In the absence of a strong scatterer, equation (7) simplifies to the widely used Rayleigh model. 


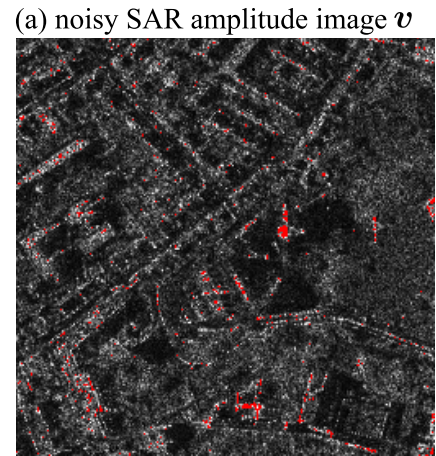

(b) TV denoising

(c) image decomposition: bounded variations $\boldsymbol{u}_{\mathrm{BV}}$ and sparse $\boldsymbol{u}_{\mathrm{S}}$ parts
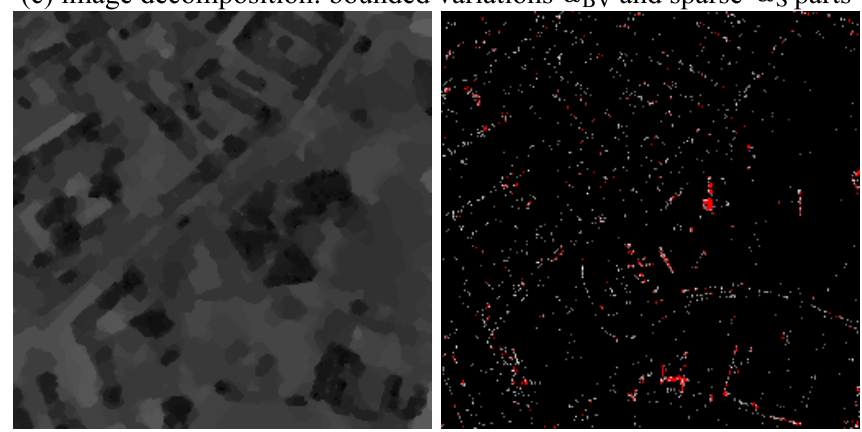

Fig. 3. SAR image denoising: comparison of TV denoising and TV+L0 decomposition (SAR image (C)CNES/DGA).

\subsection{Energy minimization}

Rice neg-log-likelihood defined in equation (7) is quasiconvex (but non-convex) in each variable. For a given value of $\boldsymbol{u}_{\mathrm{BV}}$, it reaches its unique minimum at $\boldsymbol{u}_{\mathrm{S}}^{*}\left(\boldsymbol{u}_{\mathrm{BV}}\right)$ (red curve on Fig. 2). At each pixel $k$, and for each value $\left\{\alpha_{1}, \alpha_{2}, \cdots, \alpha_{m_{\mathrm{BV}}}\right\}$, the corresponding value of $u_{\mathrm{S} k}{ }^{*}\left(u_{\mathrm{BV} k}\right)$ can be computed efficiently by bisection. The number of quantization levels $m_{\mathrm{S}}$ of the sparse component can be chosen large to account for the high dynamic range of strong scatterers with very little overhead. The bounded variations component has very low dynamic range and choosing $m_{\mathrm{BV}} \approx$ 100 is often enough in practice. The positivity constraints $\left(\forall k, u_{\mathrm{BV} k}>0\right.$ and $\left.u_{\mathrm{S} k} \geq 0\right)$ are straightforwardly enforced by considering discrete levels that satisfy the constraints. The tuning of parameters $\beta_{\mathrm{BV}}$ and $\beta_{\mathrm{S}}$ is done by hand by first setting a suitable $\beta_{\mathrm{BV}}$ value with $\beta_{\mathrm{S}}=0$, and then choosing a value of $\beta_{\mathrm{S}}$ that gives a satisfying number of strong scatterers.

\subsection{Decomposition results}

We illustrate the method on a real SAR image (Fig. 3.a: south of Toulouse city, France, (C)CNES/DGA). Fig. 3 compares the results obtained when denoising with a total variation minimization approach and with the TV+L0 decomposition approach. The TV approach corresponds to setting $\beta_{\mathrm{S}}$ to infinity. Strong scatterers are either suppressed or distorted (reduced amplitude, spreading) as they do not fit to the prior model (result of TV denoising shown Fig. 3.b). TV+L0 de- composition produces much smoother regions in the BV component (Fig. 3.c left) and sharp targets in the sparse component (Fig. 3.c right).

\section{CONCLUSION}

The proposed decomposition provides a better model to images such as in SAR imaging (both in terms of scene prior and noise model). The challenging highly non-convex problem can be exactly solved by computation of a min-cut. Though the number of quantization levels can be limited, the graphbased minimization algorithm is limited in its use by the cost of the memory representation of the graph: $N \times m_{\mathrm{BV}}$ nodes and about $3 N \times m_{\mathrm{BV}}$ pairs of arcs.

\section{REFERENCES}

[1] L. Rudin, S. Osher, and E. Fatemi, "Nonlinear total variation based noise removal algorithms," Physica D., vol. 60, pp. 259-268, 1992.

[2] A. Buades, B. Coll, and J. Morel, "The staircasing effect in neighborhood filters and its solution," IEEE TIP, vol. 15, no. 6, pp. 1499-1505, 2006.

[3] Y. Meyer, Oscillating patterns in image processing and nonlinear evolution equations: the fifteenth Dean Jacqueline B. Lewis memorial lectures, AMS, 2001.

[4] J.F. Aujol, G. Gilboa, T. Chan, and S. Osher, "Structuretexture image decomposition: modeling, algorithms, and parameter selection," IJCV, vol. 67, no. 1, 2006.

[5] W. Yin, D. Goldfarb, and S. Osher, "Image CartoonTexture Decomposition and Feature Selection Using the Total Variation Regularized $L^{1}$ Functional," LNCS, vol. 3752, pp. 73, 2005.

[6] M. Elad, J.L. Starck, P. Querre, and DL Donoho, "Simultaneous cartoon and texture image inpainting using morphological component analysis (MCA)," App. Comp. Harm. Anal., vol. 19, no. 3, pp. 340-358, 2005.

[7] J. Gilles, "Image Decomposition: Theory, Numerical Schemes, and Performance Evaluation," Adv. in Imag. Electron Phys., vol. 158, pp. 89-137, 2009.

[8] H. Ishikawa, "Exact optimization for Markov random fields with convex priors," IEEE TPAMI, vol. 25, no. 10, pp. 1333-1336, 2003.

[9] J. Darbon and M. Sigelle, "Image restoration with discrete constrained Total Variation part II: Levelable functions, convex priors and non-convex cases," J. Math. Im. Vis., vol. 26, no. 3, pp. 277-291, 2006.

[10] J.W. Goodman, Speckle phenomena in optics: theory and applications, Roberts\& Co Publishers, 2006. 